\title{
Interactions between incentive valence and action information in a cued approach-avoidance task
}

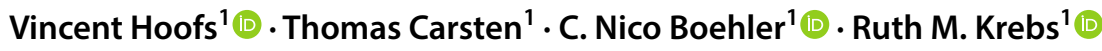

Received: 5 July 2017 / Accepted: 2 January 2018

๑) Springer-Verlag GmbH Germany, part of Springer Nature 2018

\begin{abstract}
Environmental stimuli can provoke specific response tendencies depending on their incentive valence. While some studies report positive-approach and negative-avoidance biases, others find no such mappings. To further illuminate the relationship between incentive valence and action requirement, we combined a cued monetary incentive paradigm with an approach/ avoidance joystick task. Incentive type was manipulated between groups: The reward group won money, while the punishment group avoided losing money for correct and fast responses to targets following incentive cues. Depending on their orientations, targets had to be 'approached' or 'avoided'. Importantly, incentive valence (signaled by cue color) was orthogonal to action requirement (target orientation). Moreover, targets could carry valence-associated information or not (target color), which was, however, task-irrelevant. First, we observed that both valence cues (reward/punishment) improved performance compared to neutral cues, independent of the required action (approach/avoid), suggesting that advance valence cues do not necessarily produce specific action biases. Second, task-irrelevant valence associations with targets promoted action biases, with valence-associated targets facilitating approach and impairing avoid responses. Importantly, this approach bias for valence-associated targets was observed in both groups and hence occurred independently of absolute valence ('unsigned'). This rather unexpected finding might be related to the absence of a direct contrast between positive valence and negative valence within groups and the common goal to respond fast and accurately in all incentive trials. Together, our results seem to challenge the notion that monetary incentives trigger 'hard-wired' valence-action biases in that specific design choices seem to modulate the presence and/or direction of valence-action biases.
\end{abstract}

Keywords Reward $\cdot$ Punishment $\cdot$ Valence $\cdot$ Action $\cdot$ Approach/avoidance $\cdot$ Monetary incentive paradigm

\section{Introduction}

To make optimal decisions when facing several possible courses of action, people have to recognize environmental stimuli as positive or negative, and to decide how to respond to them appropriately. Theoretical accounts assume that at least two systems are involved in the regulation of behavior in response to different kinds of stimuli (e.g., Konorski, 1967; Thayer, 1989). Specifically, the so-called Behavioral Activation System would be concerned with approaching appetitive stimuli, whereas the Behavioral Inhibition System deals with withdrawal or avoidance of aversive stimuli (Carver \& Scheier, 1998; Gray, 1990). As such, these two

Vincent Hoofs

vincent.hoofs@ugent.be

1 Department of Experimental Psychology, Ghent University, Henri Dunantlaan 2, 9000 Ghent, Belgium systems are assumed to induce behavioral facilitation when a person is required to either approach positive stimuli, or avoid negative stimuli.

In previous studies, it was already shown that natural valence-action biases can be probed in an experimental context by manipulating the attractiveness of stimuli (i.e., valence) and action requirement in an orthogonal fashion. For instance, in an early behavioral study, participants were faster when they were instructed to either pull cards with a positive word meaning towards them or to push cards with a negative word meaning away. In contrast, participants were slower when being instructed to pull negative cards towards them or to push positive cards away (Solarz, 1960). These valence-action biases for stimuli with an inherent emotional content are considered to reflect fairly automatic, hard-wired motivational tendencies, which have proven beneficial for survival (Bargh \& Chartrand, 1999; Zajonc, 1980). 
To date, numerous studies have described similar biases using inherent emotional content in animated approach/avoidance computer tasks (Chen \& Bargh, 1999; Krieglmeyer, Deutsch, De Houwer, \& De Raedt, 2010; Neumann, Förster, \& Strack, 2003; Phaf, Mohr, Rotteveel, \& Wicherts, 2014; Seibt, Neumann, Nussinson, \& Strack, 2008). For example, in a set of experiments conducted by Krieglmeyer et al. (2010), participants responded to a central emotion word by moving a manikin (representing the 'self') up and down on the screen using two separate buttons. In one block, positive words required upward movements and negative words required downward movements, while the reversed mapping was applied in the other block. In addition, the initial location of the manikin changed from trial to trial (below or above the word), so that the approach/avoidance dimension could be dissociated from the physical movement direction. In brief, the authors found valence-action biases, i.e., faster distance decreases between the imagined self and positive words when approaching these, and faster distance increases when avoiding negative words. Interestingly, these compatibility effects were observed regardless of the initial manikin location and physical movement direction (i.e., arm flexion or extension), which suggests a fairly automatic activation of approach and avoidance action codes for inherently positive and negative events, respectively (Krieglmeyer et al., 2010; Markman \& Brendl, 2005).

In addition to these studies which applied inherent emotional content, an independent, but clearly related line of research and theorizing has described similar valence-action biases in the context of reinforcement learning, considering both Pavlovian and instrumental learning (Dayan, Niv, Seymour, \& Daw, 2006; Geurts, Huys, Den Ouden, \& Cools, 2013; Huys et al., 2010). While the observed biases for stimuli with newly acquired valence are very similar to the ones found for inherently emotional stimulus material, this research focuses on how these associations have been established or learned in the first place. One important observation is that over-learned, automatic Pavlovian responses to appetitive and aversive stimuli can interfere with or overrule instrumental action-outcome contingencies (Bouton, 1993, 2007; Guitart-Masip, Duzel, Dolan, \& Dayan, 2014), which highlights that once established, valence-action associations are hard to overcome-just like the ones observed for inherent emotional material.

Other studies demonstrated the occurrence of valence-action biases when using monetary incentives (reward and punishment) as appetitive and aversive events (Freeman, Razhas, \& Aron, 2014; Guitart-Masip et al., 2012; Huys et al., 2011; Wagenbreth et al., 2015). For example, Guirtart-Masip et al. (2012) developed a Go/noGo paradigm in which participants learned to associate different cues (fractal images) with certain combinations of incentive valence (reward vs. punishment) and actions (Go vs.
noGo) in a trial-and-error fashion. Importantly, these mappings could be compatible or incompatible with 'natural' valence-action biases (Go response to win money/noGo response to avoid losing money vs. noGo response to win money/Go response to avoid losing money). In this paradigm, significantly increased accuracy rates were observed for compatible responses (Go-to-win and noGo-to-avoid-losing) compared to incompatible ones (noGo-to-win and Goto-avoid-losing), confirming that learned incentive value signals can produce similar valence-action biases as emotional stimuli. Other studies which employed similar versions of this Go/noGo paradigm to study the effects of action and valence also found such valence-action couplings. These studies report significantly higher accuracy rates for all possible compatible valence-action mappings (i.e., Go-to-win vs. Go-to-avoid-losing and noGo-to-avoid-losing vs. noGoto-win; Richter et al. 2014), or at least for some selected compatible mappings (Cavanagh, Eisenberg, Guitart-Masip, Huys, \& Frank, 2013; Guitart-Masip et al., 2011). From the above observations, it could be concluded that valence and action dimensions are mutually dependent, with a fairly automatic tendency to approach reward-related and avoid punishment-related stimuli-which parallels approach/ avoidance tendencies to emotional material. Interestingly, as a result of this mutual dependency, several studies aimed to influence subjective incentive valence towards specific unhealthy stimuli by instructing participants to perform an avoid response when being presented with such a stimulus (e.g., Becker, Jostmann, Wiers, \& Holland, 2015; Kong et al., 2015).

In contrast to these observations, other studies have found no such biases, i.e., no difference in task performance between reward and punishment manipulations. For example, in a study employing a selective stop-change task performed by Verbruggen and McLaren (2016), which probes inhibitory control processes, no differences were observed in performance between a participant group that could earn rewards for correct task performance and one which could avoid losing money for incorrect performance. This finding seems rather inconsistent with the notion reported above of a natural mapping between reward-approach and punishmentavoidance (cf. Guitart-Masip et al., 2012), in that one would expect that response inhibition would be facilitated in the context of negative valence (here, monetary loss), while response execution would be facilitated in the context of positive valence (here, monetary win). Earlier studies made a similar observation in the visual attention domain (Engelmann, Damaraju, Padmala, \& Pessoa, 2009; Engelmann \& Pessoa, 2007; Small et al., 2005). Without going into much detail, these studies showed that both reward and punishment manipulations lead to similar performance improvements in the form of faster response execution in a forced choice task. Now, to relate these observations back to the 
before-mentioned studies observing valence-action biases, we need to consider important paradigmatic differences, which might explain these diverging findings. One difference between studies reporting valence-action biases and those that do not is that reward and punishment information in the latter are not conveyed by unique stimuli, but in a contextual fashion (trial or block cues), which likely promotes a more controlled, strategic approach to maximize monetary incentives. Moreover, in the before-mentioned studies that did not observed clear valence-action biases (including Engelmann et al., 2009; Verbruggen \& McLaren, 2016), response requirements were orthogonal to the valence information (involving neither clear approach nor avoidance behavior), which is in contrast to the fixed valence-action mappings that are often used in a reinforcement learning context. Related to this, valence information in the latter studies was merely instructed rather than learned over time. Finally, it is also possible that these studies failed to observe such biases, because the required action was a simple button press (or the inhibition of a button press), which might be considered as a relatively weak representation of approach/ avoidance behavior.

With the present study, we aimed to bridge between different types of paradigms to test under which circumstances putative valence-action biases can occur, thereby also illuminating the reasons for seemingly inconsistent findings of earlier studies. To do so, we employed a novel cued monetary incentive approach/avoidance task that manipulates valence and action in an orthogonal fashion. More specifically, we wanted to investigate whether valence-action biases can be observed when valence and action information were separated in time and provided in an orthogonal fashion while using an intuitive approach/avoidance manipulation (i.e., an animated joystick task) that closely matches studies in the emotional domain that observed robust valence-action biases (e.g., Krieglmeyer et al., 2010). A second, and more exploratory, aim of the present study was to test whether coincidental, but entirely irrelevant incentive valence information would trigger valence-action biases, assuming that a putative hard-wired bias would occur independently of goal-directed processes. Specifically, previous studies have reported that task-irrelevant valence-related features can influence performance, either when these occur in another stimulus dimension within the same task (e.g., Krebs, Boehler, Egner, \& Woldorff, 2011; Krebs, Boehler, \& Woldorff, 2010 ) or in an entirely independent subsequent task (e.g., Anderson, Folk, Garrison, \& Rogers, 2016; Anderson, Laurent, \& Yantis, 2011; Anderson \& Yantis, 2013; Lee \& Shomstein, 2014; Theeuwes \& Belopolsky, 2012). Moreover, related designs using approach/avoidance manipulations to measure the strength of dispositions towards unhealthy stimuli (e.g., Wiers, Rinck, Dictus, \& van den Wildenberg, 2009) and to reduce such tendencies (e.g., Becker et al.,
2015) revealed that responding to task-irrelevant valence features can possibly provoke valence-action biases as well (see also De Houwer, 2003). Based on these studies, we expect that task-irrelevant positive and negative incentive signals can affect responses and potentially promote valence-action biases.

To this end, we combined a cued monetary incentive paradigm with an approach/avoidance joystick task, and manipulated incentive valence between two groups of participants. The reward group won money, while the punishment group prevented losing money by correct and fast responses in incentive compared to no-incentive trials. Importantly, incentive valence was signaled by the cue color (incentive vs. no incentive), while response requirement (push vs. pull the joystick) was indicated by the orientation of the subsequent target (i.e., response requirement was orthogonal to incentive information). In contrast to the previous studies, this design entails a separation between the presentation of valence information (in the cue) and action information (in the target). Moreover, the target could be independently drawn in a color which was either associated with incentives or not, but importantly, target color was entirely taskirrelevant and never predictive of the outcome. As such, participants in the reward group were presented with reward cues and reward-associated targets, and participants in the punishment group were presented with punishment cues and punishment-associated targets, which were contrasted with neutral, no-incentive conditions in both groups. In this way, cue color was predictive of the possibility of earning or avoiding losing money, while target color was not.

Based on the partly opposing observations of the studies mentioned above, we can generate several hypotheses with respect to our main research question: Considering that incentive valence is signaled by advance cues (i.e., orthogonal to action information) and that the common goal is to respond fast and accurately, it seems likely that both reward and punishment cues would facilitate performance in a strategic, goal-directed manner, independent of the required action. Alternatively, since we are probing a more intuitive representation of approach/avoidance behavior (animated joystick task) to match studies that found robust valence-action mappings in the emotional domain, we might observe differential effects of reward and punishment cues in approach and avoid trials-even if valence and action signals are separated in time. Regarding our second, more exploratory research question, we expect that task-irrelevant but coincidental valence associations with the target would trigger valence-action biases, as reflected in facilitation of approach responses in the reward group and facilitation of avoid responses in the punishment group. To test these hypotheses, the main statistical tests for both research questions (cue valence and target-valence associations) are the interactions between valence, response type, and group. 


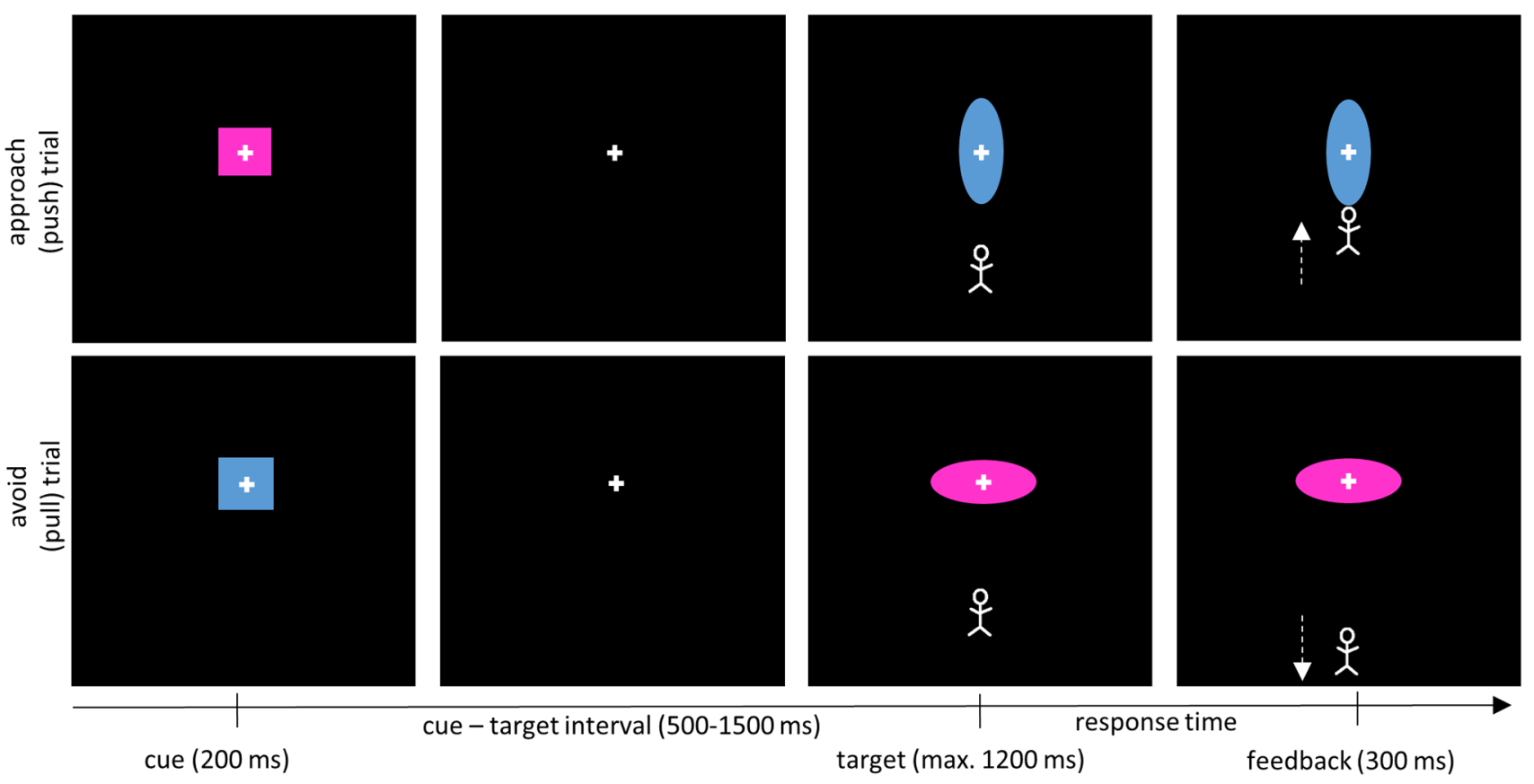

Fig. 1 Schematic trial overview. Participants were presented with color cues (square). One of the cue colors (blue/pink) indicated reward prospect (reward group) or punishment prospect (punishment group), while the other color indicated the absence of reward or punishment prospect (neutral cue). After a variable interval, a manikin appeared together with the target (ellipse), and participants were instructed to respond to the orientation of the target (vertical $=$ push/ approach, horizontal $=$ pull/avoid) as fast as possible. The target could

Specifically, while a three-way interaction between these factors would provide evidence for a 'signed' valence-action bias (opposite direction for reward and punishment), a twoway interaction between valence and response type across groups would index an 'unsigned' bias (same direction for reward and punishment). Finally, the absence of such interactions would suggest the absence of a valence-action bias. To preempt the results, we did not find any indication for valence-action biases for advance incentive valence cues (neither signed nor unsigned), but observed an unsigned bias for coincidental incentive valence associations with the target.

\section{Methods}

\section{Participants}

Eighty participants took part in the present study. One half was assigned to the reward group ( $n=40,29$ females, mean age \pm SD $22.1 \pm 2.8$, age range 18-29), and the other half to the punishment group $(n=39,28$ females, mean age \pm SD $20.9 \pm 2.5$, age range 18-28). Data from one participant (punishment group) were excluded due to a large amount be independently drawn in blue or pink as well, but target color was entirely task-irrelevant. Correct in-time responses were followed by a response-congruent movement of the manikin. The figure illustrates two correct trials, with dotted arrows serving as illustration (not presented during the experiment). Incorrect or too late responses were followed by a black screen showing a white cross or clock, respectively

of too late responses ( $>35 \%)$. The initial inclusion criteria were age between 18 and 35 years, right-handedness, normal color perception, normal or corrected-to-normal vision, and no (history of) diagnosed mental disorders. The study was approved by the local ethics board and written informed consent was obtained from all participants in advance of participation. All procedures were in accordance with the Declaration of Helsinki from 1964 and its later amendments. Participants received 5 euro as base reimbursement for 30 min plus an average bonus of 2.40 euro in the reward group, and 2.43 euro in the punishment group.

\section{Paradigm and procedure}

Participants performed an approach/avoidance task in which push and pull responses were administered by means of a joystick (Fig. 1). A small white fixation cross was maintained in the upper half of a black screen throughout the experiment. In each trial, participants first saw a colored cue (square, $200 \mathrm{~ms}$ ) indicating reward or punishment prospect, which was followed by the presentation of a small manikin and a target stimulus (ellipse) after a variable interval (500-1500 ms). Participants were instructed to respond to the orientation of this target stimulus, which could be either 
oriented vertically (requiring a push response) or horizontally (requiring a pull response), and which was presented until the response was made, or for maximal $1200 \mathrm{~ms}$. When responding correct and fast enough, the manikin animation moved in the selected direction on the screen for $300 \mathrm{~ms}$, serving as 'correct' feedback. When responding incorrectly or too late, the target disappeared and participants saw a white colored cross or a clock image for $300 \mathrm{~ms}$, serving as 'incorrect' and 'too late' feedback, respectively. After a variable interval of $1500-3000 \mathrm{~ms}$, the next trial was presented.

While one of the two cue colors (i.e., blue or pink) was predicting reward prospect in the reward group or punishment prospect in the punishment group, the other color indicated neutral, no-incentive trials. Cue color-valence mappings were counterbalanced across participants within each group. In the reward group, participants were informed that they would start with 0 euro beyond the base compensation, and that they could win bonus money during the experiment, while in the punishment group, participants were informed that they would start with 3 euro, and that they should try to avoid losing money during the experiment. When responding correct and fast enough to a target stimulus that was preceded by a reward cue (reward group), participants earned a reward of 2 cents. Slow or incorrect responses after reward cues, as well as responses following neutral cues did not affect the monetary outcome. In contrast, when participants responded slow or incorrect to a target preceded by a punishment cue (punishment group), they lost 2 cents. Correct and in-time responses after punishment cues as well as responses following neutral cues did not affect the monetary outcome. To motivate all participants to perform the experiment in a similar way, a staircase procedure was applied, which dynamically adjusted the response deadline on the basis of participants' individual performance (Cornsweet, 1962). This procedure was set to yield $80 \%$ correct feedback per experimental condition. ${ }^{1}$ Importantly, all responses in a fixed window between 150 and $1200 \mathrm{~ms}$ after target onset were

\footnotetext{
${ }^{1}$ For the first 20 participants of the reward group, a single dynamically adjusted response interval was applied across all conditions. To prevent biasing of specific conditions by presenting participants with lower rates of correct feedback for conditions with higher average RTs, we changed the program to apply condition-specific response intervals in the rest of the experiment. Statistical testing (one-staircase vs. condition-specific staircases) revealed only a marginal significant Cue valence $\times$ Target valence association $\times$ Staircase procedure interaction in the RT data: $F(1,38)=3.41, p=.073 ; \eta^{2}{ }_{\mathrm{p}}=.082$, indicating that this interaction was weaker for the one-staircase procedure: $F(1,19)=0.99, p=.33 ; \eta^{2}=.049$ than for the conditionspecific procedure: $F(1,19)=13.07, p=.002 ; \eta_{\mathrm{p}}^{2}=.407$. Furthermore, no differences between the first 20 participants (one-staircase) and the second 20 participants (condition-specific staircases) within the win-valence group were observed in the RT or error rate data (all $p>.199$ ).
}

considered for the analysis-regardless of the staircasing procedure.

To get familiar with the experiment and the different cue color meanings, participants started with a short training sequence of eight trials. Afterwards, three experimental blocks of 104 trials were performed, which were separated from each other by short breaks. During these breaks, participants were informed about their accuracy (based on the feedback provided to them during the experiment) and the total amount of money earned or preserved so far.

The number of trials was equally distributed over eight conditions. Conditions were formed by combinations of the factors Cue valence (cue color), Target valence association (target color), and Response type (target orientation). Cue colors signaled reward/punishment prospect (reward/punishment cue vs. neutral cue), target colors could be reward/ punishment-associated or not, and the response type could be push ('approach', vertical target) or pull ('avoid', horizontal target). Importantly, only the cue color was actually predicting reward/punishment prospect, while target color was entirely task-irrelevant. This information was made explicit to the participants.

\section{Data analyses}

RTs (correct trials only), response error rates (in-time responses), and inverse efficiency scores (RT divided by proportion of correct responses, cf. Bruyer and Brysbaert 2011) were submitted to a $2 \times 2 \times 2$ repeated-measures analysis of variance (rANOVA) with factors Cue valence (valence/ non-valence cue), Target valence association (valenceassociated/non-valence-associated target) and Response type (approach/avoidance) as within-subject factors, and Group (reward group/punishment group) as between-subject factor. Note that in these analyses, the factor Cue valence reflects reward in the reward group and punishment in punishment group (the same logic applies to the factor Target valence association). As noted above, the main focus in the analysis is to test for interactions between Cue valence, Response type, and Group, as well as between Target valence association, Response type, and Group. Other interactions and main effects are reported and discussed for a comprehensive overview of the data pattern.

\section{Results}

\section{Response times (RTs)}

Mean RTs are depicted in Fig. 2. Responses in the reward group were globally faster than those of the punishment group (main effect of Group: $F(1,77)=4.94, p=.029$; $\eta_{\mathrm{p}}^{2}=.060$ ), and approach (push) responses were globally 

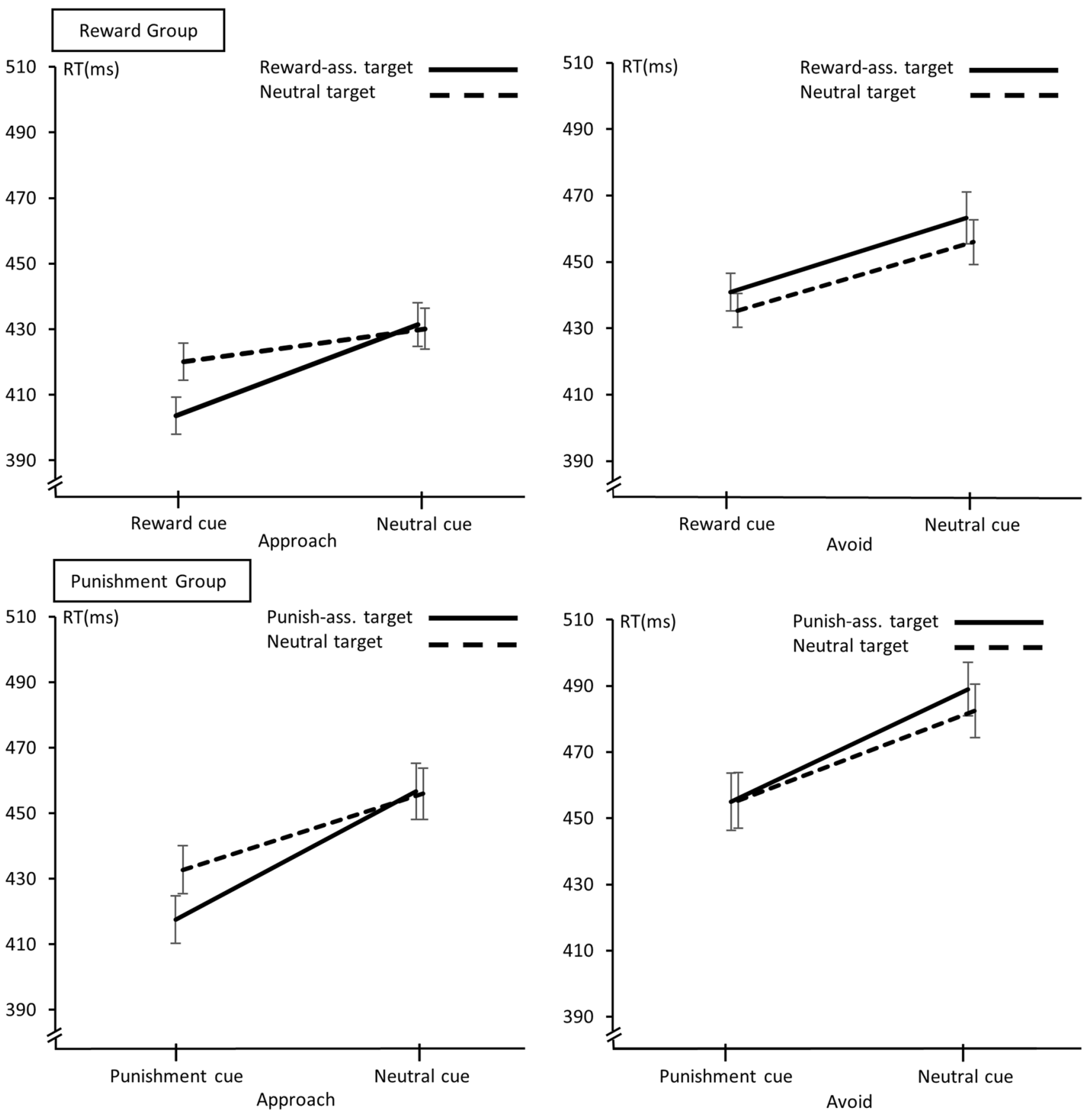

Fig. 2 RT results. The figure presents the mean RTs for approach responses (left) and avoid responses (right) for the reward group (top) and the punishment group (bottom). Error bars indicate SE of the means

faster than avoid (pull) responses (main effect of Response type: $\left.F(1,77)=141.48, p<.001 ; \eta_{\mathrm{p}}^{2}=.648\right)$. Moreover, valence cues led to faster responses as compared to neutral cues (main effect of Cue valence: $F(1,77)=121.34, p<.001$; $\eta_{\mathrm{p}}^{2}=.612$ ), and this facilitation was stronger in the punishment compared to the reward group (Cue valence $\times$ Group: $\left.F(1,77)=5.29, p=.024 ; \eta_{\mathrm{p}}^{2}=.064\right)$. Moreover, Cue valence interacted with Target valence association: $F(1$, $77)=22.93, p<.001 ; \eta_{\mathrm{p}}^{2}=.229$, reflecting that the overall facilitation elicited by valence cues was further enhanced by the presence of valence-associated targets across groups. Regarding the main research question, concerning the effect of cue valence on approach/avoid responses, we did not find evidence for valence-action biases (Cue valence $\times$ Response type $\times$ Group: $F(1,77)=0.42, p=.521$; $\eta_{p}^{2}=.005$; Cue valence $\times$ Response type: $F(1,77)=0.15$, $\left.p=.7 ; \eta_{\mathrm{p}}^{2}=.002\right)$. Rather, reward and punishment cues affected both approach and avoid trials in a similar manner. 
With regard to the second research question, concerning the effect of target valence associations, we observed an interaction between Target valence association and Response type: $F(1,77)=25.26, p<.001 ; \eta_{\mathrm{p}}^{2}=.247$. Specifically, we found that valence-associated targets (as compared to neutral ones) led to response facilitation in approach trials: $t(78)=-6.02$, $p<.001$, but to response slowing in avoid trials: $t(78)=2.64$, $p=.010$. Importantly, however, this pattern did not differ between groups (Target valence association $\times$ Response type $\times$ Group: $\left.F(1,77)=0.61, p=.436 ; \eta_{p}^{2}=.008\right)$. Finally, a three-way interaction was observed between Cue valence $\times$ Target valence association $\times$ Response type: $F(1$, $77)=7.35, p=.008 ; \eta_{\mathrm{p}}^{2}=.087$, which was due to a differential interaction between Cue valence and Target valence association in approach $(F(1,77)=26.94, p<.001$; $\left.\eta_{\mathrm{p}}^{2}=.259\right)$ as compared to avoid responses $(F(1,77)=1.85$, $\left.p=.177 ; \eta_{\mathrm{p}}^{2}=.024\right)$, reflecting that additional facilitation by valence-associated targets following valence cues was only observed in approach trials (Fig. 2). All remaining effects did not reach significance (all $p>.181$ ).

To summarize the main findings regarding the effects of Cue valence and Target valence association, we calculated RT difference values (incentive minus neutral trials) for each factor separately (Fig. 4a). These plots illustrate that incentive cues (grey bars) affect both action types in form of a global performance benefit (neither signed nor unsigned valence-action bias), and that valence-associated targets (black bars) modulate approach and avoid responses differently (benefit approach, impair avoid), but regardless of absolute valence (unsigned valence-action bias). Note that these plots merely serve to illustrate the main effects of the two incentive factors in relation to Response type and Group.

\section{Error rates}

Mean error rates are depicted in Fig. 3. Error rates were slightly lower in the punishment compared to the reward group (trend for a main effect of Group: $F(1,77)=3.72$, $\left.p=.057 ; \eta_{\mathrm{p}}^{2}=.046\right)$, indexing a performance benefit that is in opposite direction compared to the RT data. Like in the RT data, performance was improved after valence cues (main effect of Cue valence: $F(1,77)=13.81, p<.001 ; \eta_{\mathrm{p}}^{2}=.151$ ). Again, there was no indication for a valence-action bias (Cue valence $\times$ Response type $\times$ Group: $F(1,77)=1.18$, $p=.281 ; \eta_{\mathrm{p}}^{2}=.015$; Cue valence $\times$ Response type: $F(1$, $\left.77)=1.07, p=.304 ; \eta_{\mathrm{p}}^{2}=.014\right)$, indicating that reward and punishment cues affected both approach and avoid responses in a similar way. Regarding the effects of valence-associated targets, the groups differed at trend level (Target valence association $\times$ Group: $\left.F(1,77)=2.79, p=.099 ; \eta_{\mathrm{p}}^{2}=.035\right)$, which is the consequence of a slight error reduction for punishment-associated targets compared to a slight error increase for reward-associated targets. Similar to the RT data, Target valence association interacted with Response type: $F(1,77)=26.32, p<.001 ; \eta_{\mathrm{p}}^{2}=.243$, indicating that valence-associated targets (compared to neutral ones) led to error reduction in approach trials: $t(78)=-3.62, p<.001$, and error increase in avoid trials: $t(78)=3.61, p<.001$. Finally, we found a significant three-way interaction of Target valence association $\times$ Response type $\times$ Group: $F(1$, $77)=5.07, p=.027 ; \eta_{\mathrm{p}}^{2}=.047$, indicating that valence associations carried by the target facilitated approach and impaired avoid responses stronger in the reward group $[F(1$, $\left.\left.39)=22.51, p<.001 ; \eta_{\mathrm{p}}^{2}=.366\right)\right]$ an in the punishment group $\left[F(1,38)=5.32, p=.027 ; \eta_{p}^{2}=.123\right.$; Fig. 3], suggesting that the reward-approach mapping might overall be more robust. All remaining effects did not reach significance (all $p>.135$ ).

Effects of Cue valence and Target valence association are summarized by calculating error rate difference values (incentive minus neutral trials) for both factors separately (Fig. 4b). While incentive cues (grey bars) promote a general performance benefit (neither signed nor unsigned valence-action bias), valence-associated targets (black bars) affected approach and avoid responses differently (benefit approach, impair avoid). While this pattern was observed in both groups (unsigned valence-action bias), the bias was more pronounced in the reward group. Note that these plots merely serve to illustrate the main effects of the two incentive factors in relation to Response type and Group.

\section{Speed-accuracy relationship}

In addition to RT and error rate measures, an analysis of inverse efficiency scores was performed. These scores are calculated by dividing RT by the proportion of correct responses, and are used to provide an integrated performance measure that is sensitive to potential speed-accuracy tradeoffs (Bruyer \& Brysbaert, 2011). A Cue valence $\times$ Group interaction was observed: $F(1,77)=6.68, p=.012$; $\eta_{\mathrm{p}}^{2}=.080$, again indicating that the data were more strongly modulated by Cue valence information in the punishment group than in the reward group. Furthermore, the Target valence association $\times$ Group interaction was almost significant: $F(1,77)=3.96, p=.05 ; \eta_{\mathrm{p}}^{2}=.049$, confirming the slightly different impact of Target valence association in the punishment group compared to the reward group. The three-way interaction Target valence association $\times$ Response type $\times$ Group that was observed in the error rate data was present in the inverse efficiency scores as well: $F(1,77)=5.17, p=.026 ; \eta_{\mathrm{p}}^{2}=.063$, indicating that the approach bias was more pronounced in the reward group $\left.\left[F(1,39)=31.30, p<.001 ; \eta_{\mathrm{p}}^{2}=.445\right)\right]$ than in the punishment group $\left.\left[F(1,38)=6.26, p=.017 ; \eta_{\mathrm{p}}^{2}=.141\right)\right]$. Overall, when combining RT and accuracy in one score, the main result patterns are preserved, suggesting that the results are 

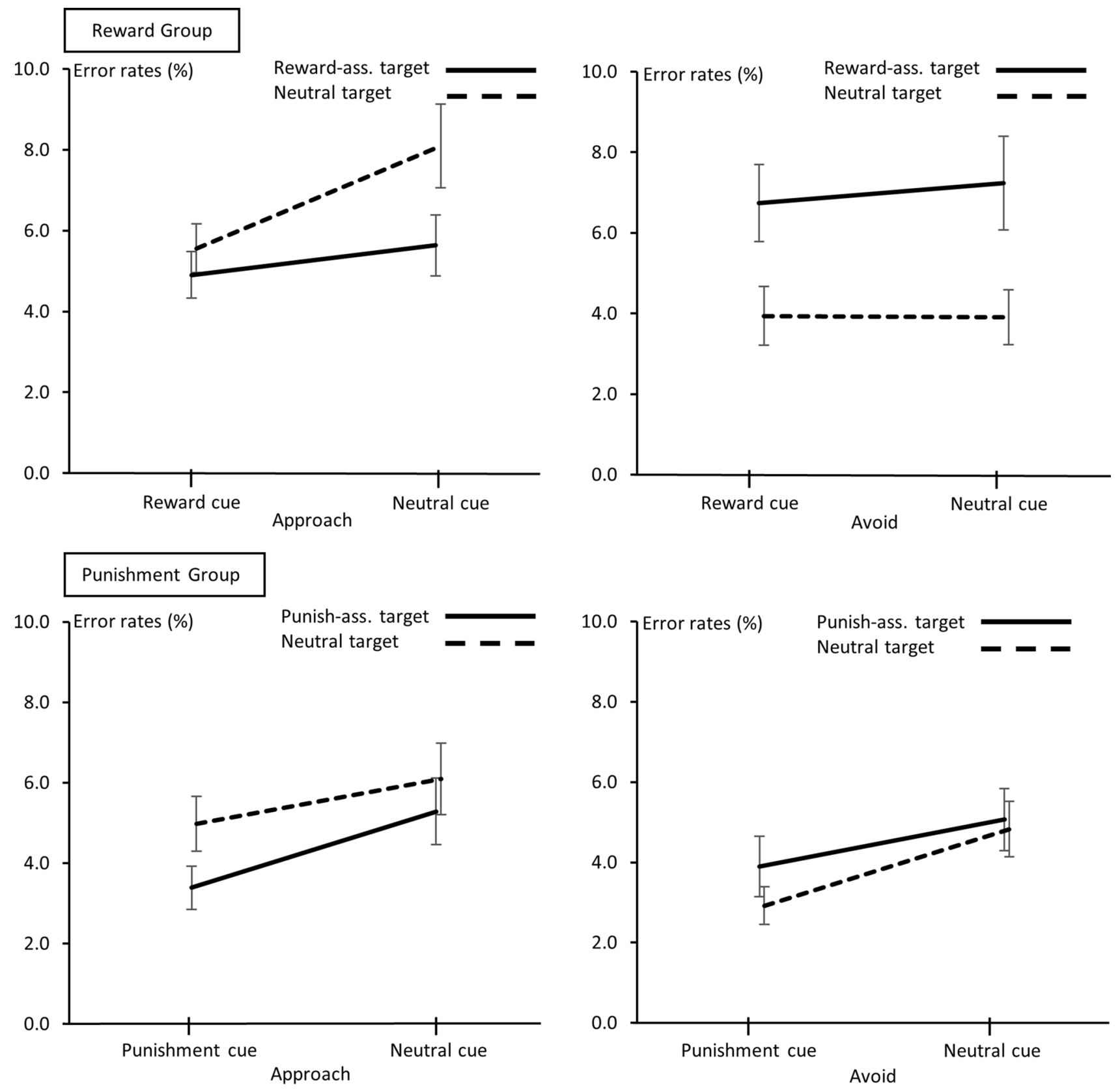

Fig. 3 Error rate results. The figure presents the mean error rates for approach responses (left) and avoid responses (right) for the reward group (top) and the punishment group (bottom). Error bars indicate SE of the means

not driven by a differential speed-accuracy trade-off in the different trial types.

\section{Discussion}

In the present study, we combined a cued monetary incentive paradigm with an approach/avoidance joystick task to study the interaction between incentive valence (betweengroup factor) and action information (within-group factor).
Importantly, in contrast to the previous studies, reward/punishment valence information (cue color) and action requirement (target orientation) were separated in time and orthogonal to one another, so that the mapping could change from trial to trial. Moreover, we used an animated joystick task with approach (push) versus avoid (pull) responses instead of button presses vs. withholding of button presses, which should be more sensitive to pick up fairly natural approach/ avoidance tendencies in response to incentive valence stimuli. Finally, on a more exploratory note, target stimuli could 

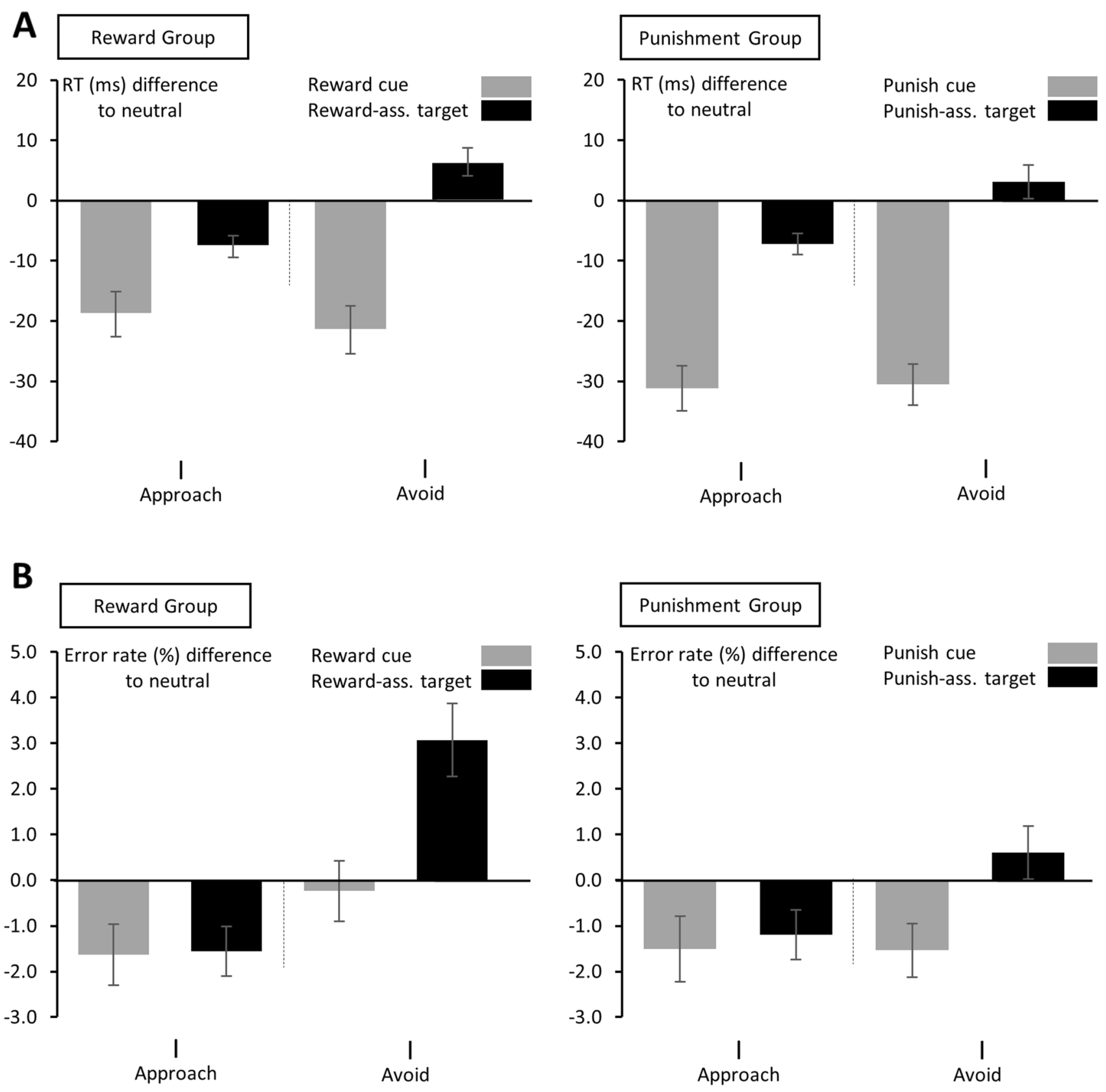

Fig. 4 Collapsed RT (a) and error rate (b) results. Difference values (incentive minus neutral) are averaged for valence cues (grey bars) and valence-associated targets (black bars) and depicted separately

carry task-irrelevant incentive valence information (target color) to test whether task-relevance is a perquisite to produce incentive valence-action biases.

The results showed that both reward and punishment cues (see grey bars in Fig. 4) improved performance with respect to the neutral conditions in the respective group, regardless of the required action (approach/avoid). In contrast, taskirrelevant valence associations carried by the target (see black bars in Fig. 4) produced an unsigned valence-action

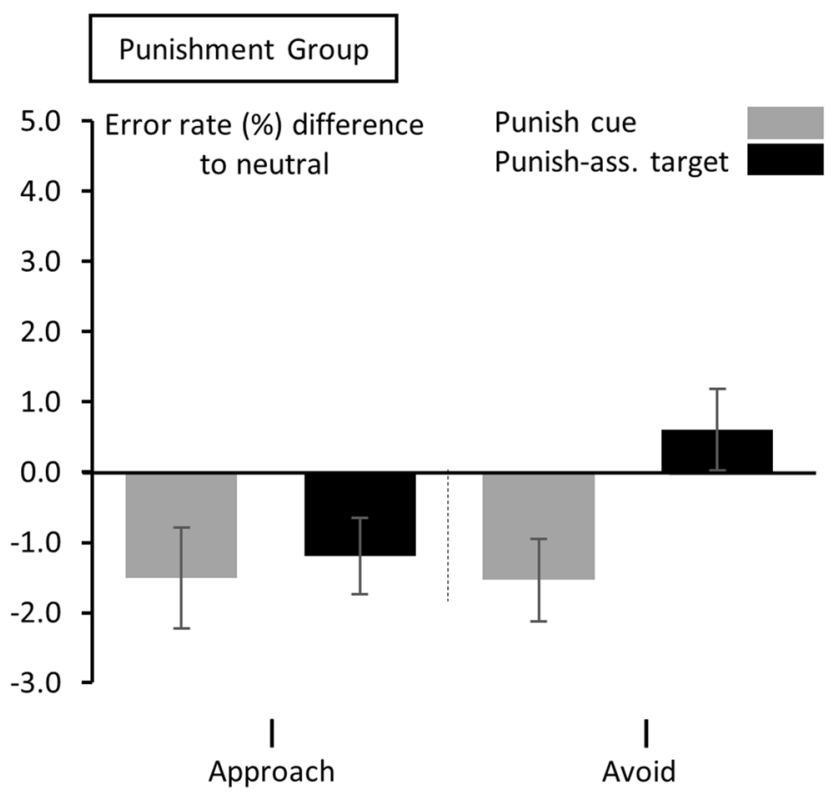

for approach/avoid responses in the two groups. Error bars indicate SE of the means

bias in both groups, with reward/punishment-associated targets leading to response facilitation in approach trials, but to performance decrements in avoid trials. This overall bias across positive and negative incentive valence is in contrast to emotional valence-action biases, where positive and negative stimuli lead to opposite action tendencies (e.g., Chen \& Bargh, 1999; Krieglmeyer et al., 2010). In sum, valence cues largely improved performance regardless of response requirements (i.e., no valence-action bias), while 
both types of valence-associated targets promoted approach and impaired avoid responses (i.e., unsigned valence-action bias). In the following, we discuss these observations in more detail with reference to the existing research.

\section{Effects of incentive cues}

First, we observed general response facilitation elicited by cue valence in both groups, as reflected in shorter response times without sacrificing response accuracy. Despite the overall similarity, this cuing effect was more pronounced in the punishment group, indicating that negative-valence cues had a stronger impact on task preparation, which might reflect an asymmetry in which loss avoidance manipulations are globally more motivating, despite of the same absolute value being at stake. This observation is generally in line with earlier work describing stronger effects for negative as compared to positive valence in different task contexts (for review, see Baumeister, Bratslavsky, Finkenauer, \& Vohs, 2001). However, others have not found significant performance differences between reward and punishment cues (e.g., Engelmann et al., 2009; Engelmann \& Pessoa, 2007; Novak \& Foti, 2015), suggesting that the global incentive cueing benefit across reward and punishment cues might be more robust, and that the additional benefit of negative incentive cues might be less reliable and dependent on the nature of the task (of note, the before-mentioned studies that did not observe differential effects all employed visual discrimination tasks). More important with regard to our research question was the observation that the incentive-based performance benefit (signaled by the cue) was independent of the required action, which was signaled to participants after a temporal delay by a different stimulus (target). The absence of valence-action biases under these circumstances suggests that these might only occur when both types of information coincide in time as was the case in the studies of Guitart-Masip et al. (2012), Cavanagh et al. (2013) and Richter et al. (2014), which all featured cues of unique valence-action mappings and found predicted valence-action biases. These paradigms are in contrast to the changing trial-by-trial combinations of valence (cue) and action (target) information in the present study. We would argue that incentive cues in the present paradigm promote more strategic, goal-directed processes, which are identical for both reward and punishment cues, rendering the occurrence of fairly automatic response biases, like observed for emotional events (Phaf et al., 2014), less likely.

Interestingly, comparable effects evoked by both reward and punishment incentives as observed in the present study have been reported by Verbruggen and McLaren (2016) using a selective stop-change task, which requires participants to execute a certain response in the majority of the Go trials, but switch to an alternative response in a subset of the trials (upon presentation of a stop-change signal). While reward and punishment prospect were manipulated in different groups like in the present study, they were active for the entire task (sustained) rather than being signaled by a cue in each trial. Compared to a control group that did not receive any incentive instructions, both incentive groups displayed behavioral modulations in the form of strategic slowing on regular Go trials and response facilitation in stop-change trials, with no indication for a difference between reward and punishment incentives (see also earlier reports by Engelmann et al., 2009; Engelmann \& Pessoa, 2007; Novak \& Foti, 2015). Together, these observations suggest that in situations in which reward and punishment are signaled by a cue or provided in a sustained fashion across blocks of trials, similar preparatory or strategic processes are triggered to obtain the task goal (i.e., 'respond fast and accurate to win or keep the money')—regardless of the type of valence, and even if the task itself can be regarded as highly sensitive to pick up natural response biases as evidenced by studies in the emotional domain (e.g., Krieglmeyer et al., 2010).

\section{Effects of task-irrelevant incentive associations with the target}

Second, task-irrelevant valence associations with the target stimulus triggered unsigned valence-action biases, in that both reward and punishment valence associations facilitated approach responses, but impaired avoid responses. Considering the reward group alone, similar reward-approach biases have been reported before in different experimental designs (Chen \& Bargh, 1999; Freeman et al., 2014; Guitart-Masip et al., 2012; Wirth, Dignath, Pfister, Kunde, \& Eder, 2016), and these seem to be comparable to biases triggered positive emotional stimuli (De Houwer, Crombez, Baeyens, \& Hermans, 2001; Solarz, 1960), as well as dispositions to approach appetitive stimuli that are considered unhealthy (e.g., Becker et al., 2015; Wiers et al., 2009). Intriguingly, and in contrast to our hypothesis, a similar approach bias for incentive valence-associated targets was observed in the punishment group. Specifically, we expected to observe an approach bias for targets associated with positive incentive valence (reward), while negative incentive valence (punishment) was expected to produce the opposite bias (with performance facilitation in avoid trials and performance decrement in approach trials), in line with the previous reports using a Go/noGo task design (e.g., Guitart-Masip et al., 2012; Richter et al., 2014). We think that the explanation for this unexpected finding is related to the composition of conditions between groups. First, while positive and negative valence stimuli were directly contrasted by GuitartMasip et al. (2012), we compared the effects of positive and negative incentive valence to a neutral baseline condition, and hence, each group only encountered one type of valence. 
It may be argued that similarities in the effects of reward and punishment manipulations are the consequence of the absence of an explicit valence contrast. While participants in the reward group may feel 'punished' when they did not earn a reward in some trials, participants in the punishment group might feel 'rewarded' when they successfully avoided a loss (Kim, Shimojo, \& O'Doherty, 2006). Second, while in the study by Guitart-Masip et al. (2012), Go and noGo responses were leading to reward and punishments with equal probability, withholding a response was never beneficial for participants in our study, thereby potentially promoting a global approach bias for all incentive trials. Considering the absence of a direct valence contrast and the emphasis on response execution in our design, it is likely that each incentive valence was coded as the one salient signal to pay attention to during the task, and to respond to fast, regardless of absolute value. That said, albeit present in both groups, the approach bias for incentive valence signals was in fact more pronounced in the reward group's error rate data, providing some evidence for a more robust mapping between approach and reward-associated target features, above and beyond largely overlapping performance modulations.

From a more general perspective, performance costs elicited by task-irrelevant valence signals (here, in avoid trials) are not unique to this study. For example, performance impairments due to task-irrelevant valence signals have been found in a rewarded Stroop task, where rewardrelated irrelevant semantic information led to stronger interference (Krebs et al., 2011, 2010), as well as in visual search tasks where spatial attention was biased towards irrelevant reward-related features (Anderson, Laurent, Yantis, Grados, \& Umaña, 2011; Anderson, Laurent, \& Yantis, 2011). In the latter studies, attentional capture triggered by stimuli that have been associated with positive (Anderson, Laurent, \& Yantis, 2011; Sali, Anderson, \& Yantis, 2014) or negative incentive valence (Müller, Rothermund, \& Wentura, 2016; Wentura, Müller, \& Rothermund, 2014) can either benefit or impair performance depending on whether the valence feature is overlapping with the target or distractor of a visual search display. Here, we observe a similar bias in the domain of action tendencies, in that targets featuring salient information are more easily approached than avoided-even if this is detrimental for the task goal.

\section{Interactions between cue and target effects, and global group differences}

In both groups, we observed that task-irrelevant valence associations with the target further augmented performance benefits induced by valence cues. This might indicate that an increase of attention during the cue-target interval led to a prioritization of any salient subsequent stimulus. Alternatively, or in addition, the observed additive effect of cue and target valence could reflect low-level feature priming effects, because the specific color of the cue is repeated in the target (Kristjánsson \& Campana, 2010; Logan, 1990; Schacter \& Buckner, 1998). In order to differentiate between unspecific attentional prioritization and specific feature priming (e.g., Grison \& Strayer, 2001; Waszak \& Hommel, 2007) in these specific conditions, one would need a paradigm in which feature repetitions are excluded by design. That said, we would not assume that the effects of target-valence associations observed in the present study could be entirely explained by attentional prioritization or feature priming due to the preceding valence cues. Most importantly, mere feature (or value) priming would predict performance benefits for feature repetitions across conditions-instead, we observe that facilitation was driven by other factors, independent of repetition. This is most evident when comparing the combination of reward cue target-reward association in approach vs. avoidance trials, which feature performance benefits and costs, respectively (Fig. 4).

In addition to trial-by-trial modulations triggered by cue valence and target-valence associations, and their interaction, the result pattern is indicative of global differences between the reward and punishment group. Specifically, when considering the RT data, there is a global offset in speed, with faster responses in the reward compared to the punishment group. Conversely, in the error rate data, we find an opposite global effect (at trend level), with fewer errors in the punishment compared to the reward group. Such context effects seem to resonate with the notion that a reward context might particularly promote fast responses, while a punishment context can promote cautious responding, which has been demonstrated in a context where punishment rate increased over time (Griffiths \& Beierholm, 2017). Notably, these global changes seem to occur more or less independently of incentive-based performance benefits from trial to trial. For example, while the reward group is faster globally (context effect), participants in the punishment group benefit more from incentive cues (trial-by-trial effect).

\section{Conclusion}

Incentive valence information in the cue operated rather strategically, and was overall beneficial in nature. Furthermore, as incentive valence information in the cue was not further affected by the required action (i.e., no valence-action bias), this seems to suggest that valence-action biases may rely on the temporal coincidence (or other dependence) between valence and action information, and that the current orthogonal design emphasizes more strategic influences of incentive cues. The influence of irrelevant valence information linked to the target itself, however, seemed to be rather automatic in nature, in that incentive signals promoted approach over 
avoid responses (even if this was not beneficial)—and this effect was fairly independent of the actual valence sign (i.e., unsigned valence-action bias). This rather unexpected finding might be related to the valence-to-neutral contrast in both groups and the common goal to respond fast in all incentive trials. As such, the results of the present study provide insights into the complex interplay between incentive valence and action signals, and seem to challenge the notion that monetary incentives trigger 'hard-wired' valence-action biases in that specific design choices seem to modulate the presence and/or direction of valence-action biases.

Funding This study was supported by a starting Grant of the European Research Council (ERC) under the Horizon 2020 framework (Grant no. 636110 awarded to RMK).

\section{Compliance with ethical standards}

Conflict of interest Vincent Hoofs, Thomas Carsten, C. Nico Boehler, and Ruth M. Krebs declare that they have no conflicts of interest.

Ethical approval All procedures performed in studies involving human participants were in accordance with the ethical standards of the institutional and/or national research committee and with the 1964 Helsinki declaration and its later amendments or comparable ethical standards.

\section{References}

Anderson, B. A., Folk, C. L., Garrison, R., \& Rogers, L. (2016). Mechanisms of habitual approach: Failure to suppress irrelevant responses evoked by previously reward-associated stimuli. Journal of Experimental Psychology: General, 145(6), 796-805. https://doi.org/10.1037/xge0000169.

Anderson, B. A., Laurent, P. A., \& Yantis, S. (2011). Value-driven attentional capture. In Proceedings of the National Academy of Sciences of the United States of America, 108(25), 10367-10371. https://doi.org/10.1073/pnas.1104047108.

Anderson, B. A., Laurent, P. A., Yantis, S., Grados, M., \& Umaña, P. (2011). Learned value magnifies salience-based attentional capture. PLoS ONE, 6(11), e27926. https://doi.org/10.1371/journal. pone.0027926.

Anderson, B. A., \& Yantis, S. (2013). Persistence of value-driven attentional capture. Journal of Experimental Psychology: Human Perception and Performance, 39(1), 6-9. https://doi.org/10.1037/ a0030860.

Bargh, J. A., \& Chartrand, T. L. (1999). The unbearable automaticity of being. American Psychologist, 54(7), 462-479. https://doi. org/10.1037/0003-066X.54.7.462.

Baumeister, R. F., Bratslavsky, E., Finkenauer, C., \& Vohs, K. D. (2001). Bad is stronger than good. Review of General Psychology, 5(4), 323-370. https://doi.org/10.1037/1089-2680.5.4.323.

Becker, D., Jostmann, N. B., Wiers, R. W., \& Holland, R. W. (2015). Approach avoidance training in the eating domain: Testing the effectiveness across three single session studies. Appetite, 85, 58-65. https://doi.org/10.1016/j.appet.2014.11.017.

Bouton, M. (2007). Learning and behavior: A contemporary synthesis. Sunderland: Sinauer Associates.

Bouton, M. E. (1993). Context, time, and memory retrieval in the interference paradigms of Pavlovian learning. Psychological Bulletin, 114(1), 80-99.
Bruyer, R., \& Brysbaert, M. (2011). Combining speed and accuracy in cognitive psychology: Is the Inverse Efficiency Score (IES) a better dependent variable than the mean Reaction Time (RT) and the Percentage of Errors (PE)? Psychologica Belgica, 51(1), 5-13. https://doi.org/10.5334/pb-51-1-5.

Carver, C. S., \& Scheier, M. (1998). On the self-regulation of behavior. Cambridge: Cambridge University Press.

Cavanagh, J. F., Eisenberg, I., Guitart-Masip, M., Huys, Q., \& Frank, M. J. (2013). Frontal theta overrides pavlovian learning biases. The Journal of Neuroscience: The Official Journal of the Society for Neuroscience, 33(19), 8541-8548. https://doi.org/10.1523/ jneurosci.5754-12.2013.

Chen, M., \& Bargh, J. A. (1999). Consequences of automatic evaluation: Immediate behavioral predispositions to approach or avoid the stimulus. Personality and Social Psychology Bulletin, 25(2), 215-224. https://doi.org/10.1177/0146167299025002007.

Cornsweet, T. N. (1962). The staircase-method in psychophysics. The American Journal of Psychology, 75(3), 485. https://doi. org/10.2307/1419876.

Dayan, P., Niv, Y., Seymour, B., \& Daw, N. D. (2006). The misbehavior of value and the discipline of the will. Neural Networks, 19(8), 1153-1160. https://doi.org/10.1016/j.neunet.2006.03.002.

De Houwer, J. (2003). A structural analysis of indirect measures of attitudes. The psychology of evaluation: Affective processes in cognition and emotion (pp. 219-244).

De Houwer, J., Crombez, G., Baeyens, F., \& Hermans, D. (2001). On the generality of the affective Simon effect. Cognition \& Emotion, 15(2), 189-206. https://doi.org/10.1080/0269993004200051.

Engelmann, J. B., Damaraju, E., Padmala, S., \& Pessoa, L. (2009). Combined effects of attention and motivation on visual task performance: Transient and sustained motivational effects. Frontiers in Human Neuroscience, 3, 4. https://doi.org/10.3389/ neuro.09.004.2009.

Engelmann, J. B., \& Pessoa, L. (2007). Motivation sharpens exogenous spatial attention. Emotion, 7(3), 668-674. https://doi. org/10.1037/1528-3542.7.3.668.

Freeman, S. M., Razhas, I., \& Aron, A. R. (2014). Top-down response suppression mitigates action tendencies triggered by a motivating stimulus. Current Biology: CB, 24(2), 212-216. https://doi. org/10.1016/j.cub.2013.12.019.

Geurts, D. E. M., Huys, Q. J. M., Ouden, D. H. E., \& Cools, R. (2013). Aversive Pavlovian Control of Instrumental Behavior in Humans. https://doi.org/10.1162/jocn_a_00425.

Gray, J. A. (1990). Brain systems that mediate both emotion and cognition. Cognition \& Emotion, 4(3), 269-288. https://doi. org/10.1080/02699939008410799.

Griffiths, B., \& Beierholm, U. R. (2017). Opposing effects of reward and punishment on human vigor. Scientific Reports, 7, 42287. https://doi.org/10.1038/srep42287.

Grison, S., \& Strayer, D. L. (2001). Negative priming and perceptual fluency: More than what meets the eye. Perception \& Psychophysics, 63(6), 1063-1071. https://doi.org/10.3758/BF03194524.

Guitart-Masip, M., Duzel, E., Dolan, R., \& Dayan, P. (2014). Action versus valence in decision making. Trends in Cognitive Sciences, 18(4), 194-202. https://doi.org/10.1016/j.tics.2014.01.003.

Guitart-Masip, M., Fuentemilla, L., Bach, D. R., Huys, Q. J. M., Dayan, P., Dolan, R. J., \& Duzel, E. (2011). Action dominates valence in anticipatory representations in the human striatum and dopaminergic midbrain. The Journal of Neuroscience: The Official Journal of the Society for Neuroscience, 31(21), 7867-7875. https://doi.org/10.1523/jneurosci.6376-10.2011.

Guitart-Masip, M., Huys, Q. J. M., Fuentemilla, L., Dayan, P., Duzel, E., \& Dolan, R. J. (2012). Go and no-go learning in reward and punishment: Interactions between affect and effect. NeuroImage, 62(1), 154-166. https://doi.org/10.1016/j. neuroimage.2012.04.024. 
Huys, Q. J. M., Cools, R., Gölzer, M., Friedel, E., Dolan, R., Heinz, A., \& Peter, D. (2010). Approaching avoidance: asymmetries in reward and punishment processing. Frontiers in Neuroscience. 4. https://doi.org/10.3389/conf.fnins.2010.03.00179.

Huys, Q. J. M., Cools, R., Gölzer, M., Friedel, E., Heinz, A., Dolan, R. J., \& Raftery, A. (2011). Disentangling the roles of approach, activation and valence in instrumental and pavlovian responding. PLoS Computational Biology, 7(4), e1002028. https://doi. org/10.1371/journal.pcbi.1002028.

Kim, H., Shimojo, S., \& O’Doherty, J. P. (2006). Is avoiding an aversive outcome rewarding? Neural substrates of avoidance learning in the human brain. PLoS Biology, 4(8), e233. https://doi. org/10.1371/journal.pbio.0040233.

Kong, G., Larsen, H., Cavallo, D. A., Becker, D., Cousijn, J., Salemink, E., \& Krishnan-Sarin, S. (2015). Re-training automatic action tendencies to approach cigarettes among adolescent smokers: a pilot study. The American Journal of Drug and Alcohol Abuse, 41(5), 425-432. https://doi.org/10.3109/00952990.2015.1049492.

Konorski, J. (1967). Integrative activity of the brain; an interdisciplinary approach. Chicago:University of Chicago Press.

Krebs, R. M., Boehler, C. N., Egner, T., \& Woldorff, M. G. (2011). The neural underpinnings of how reward associations can both guide and misguide attention. The Journal of Neuroscience: The Official Journal of the Society for Neuroscience, 31(26), 9752-9759. https://doi.org/10.1523/JNEUROSCI.0732-11.2011

Krebs, R. M., Boehler, C. N., \& Woldorff, M. G. (2010). The influence of reward associations on conflict processing in the Stroop task. Cognition, 117(3), 341-347. https://doi.org/10.1016/j. cognition.2010.08.018.

Krieglmeyer, R., Deutsch, R., De Houwer, J., \& De Raedt, R. (2010). Being moved: Valence activates approach-avoidance behavior independently of evaluation and approach-avoidance intentions. Psychological Science, 21(4), 607-613. https://doi. org/10.1177/0956797610365131.

Kristjánsson, Á, \& Campana, G. (2010). Where perception meets memory: A review of repetition priming in visual search tasks. Attention, Perception, \& Psychophysics, 72(1), 5-18. https://doi. org/10.3758/APP.72.1.5.

Lee, J., \& Shomstein, S. (2014). Reward-based transfer from bottom-up to top-down search tasks. Psychological Science, 25(2), 466-475. https://doi.org/10.1177/0956797613509284.

Logan, G. D. (1990). Repetition priming and automaticity: Common underlying mechanisms? Cognitive Psychology, 22(1), 1-35. https://doi.org/10.1016/0010-0285(90)90002-L.

Markman, A. B., \& Brendl, C. M. (2005). Constraining theories of embodied cognition. Psychological Science, 16(1), 6-10. https:// doi.org/10.1111/j.0956-7976.2005.00772.x.

Müller, S., Rothermund, K., \& Wentura, D. (2016). Relevance drives attention: Attentional bias for gain- and loss-related stimuli is driven by delayed disengagement. The Quarterly Journal of Experimental Psychology, 69(4), 752-763. https://doi.org/10.10 80/17470218.2015.1049624.

Neumann, R., Förster, J., \& Strack, F. (2003). Motor Compatibility: The Bidirectional Link Between Behavior and Evaluation. Mahwah:Lawrence Erlbaum Associates Publishers.

Novak, K. D., \& Foti, D. (2015). Teasing apart the anticipatory and consummatory processing of monetary incentives: An eventrelated potential study of reward dynamics. Psychophysiology, 52(11), 1470-1482. https://doi.org/10.1111/psyp.12504.

Phaf, R. H., Mohr, S. E., Rotteveel, M., \& Wicherts, J. M. (2014). Approach, avoidance, and affect: a meta-analysis of approach-avoidance tendencies in manual reaction time tasks. Frontiers in Psychology, 5, 378. https://doi.org/10.3389/ fpsyg.2014.00378.

Richter, A., Guitart-Masip, M., Barman, A., Libeau, C., Behnisch, G., Czerney, S., \& Schott, B. H. (2014). Valenced action/inhibition learning in humans is modulated by a genetic variant linked to dopamine D2 receptor expression. Frontiers in Systems Neuroscience, 8, 140. https://doi.org/10.3389/fnsys.2014.00140.

Sali, A. W., Anderson, B. A., \& Yantis, S. (2014). The role of reward prediction in the control of attention. Journal of Experimental Psychology. Human Perception and Performance, 40(4), 16541664. https://doi.org/10.1037/a0037267.

Schacter, D. L., \& Buckner, R. L. (1998). Priming and the Brain. Neuron, 20(2), 185-195. https://doi.org/10.1016/ S0896-6273(00)80448-1.

Seibt, B., Neumann, R., Nussinson, R., \& Strack, F. (2008). Movement direction or change in distance? Self- and object-related approachavoidance motions. Journal of Experimental Social Psychology, 44(3), 713-720. https://doi.org/10.1016/j.jesp.2007.04.013.

Small, D. M., Gitelman, D., Simmons, K., Bloise, S. M., Parrish, T., \& Mesulam, M. (2005). Monetary incentives enhance processing in brain regions mediating top-down control of attention. Cerebral Cortex, 15(12), 1855-1865. https://doi.org/10.1093/cercor/ bhi063.

Solarz, A. K. (1960). Latency of instrumental responses as a function of compatibility with the meaning of eliciting verbal signs. Journal of Experimental Psychology, 59(4), 239-245. https://doi. org/10.1037/h0047274.

Thayer, R. E. (1989). The Biopsychology of Mood and Arousal. Oxford:Oxford University Press.

Theeuwes, J., \& Belopolsky, A. V. (2012). Reward grabs the eye: Oculomotor capture by rewarding stimuli. Vision Research, 74, 80-85. https://doi.org/10.1016/j.visres.2012.07.024.

Verbruggen, F., \& McLaren, R. (2016). Effects of reward and punishment on the interaction between going and stopping in a selective stop-change task. Psychological Research Psychologische Forschung. https://doi.org/10.1007/s00426-016-0827-5.

Wagenbreth, C., Zaehle, T., Galazky, I., Voges, J., Guitart-Masip, M., Heinze, H.-J., \& Düzel, E. (2015). Deep brain stimulation of the subthalamic nucleus modulates reward processing and action selection in Parkinson patients. Journal of Neurology, 262(6), 1541-1547. https://doi.org/10.1007/s00415-015-7749-9.

Waszak, F., \& Hommel, B. (2007). The costs and benefits of crosstask priming. Memory \& Cognition, 35(5), 1175-1186. https:// doi.org/10.3758/BF03193487.

Wentura, D., Müller, P., \& Rothermund, K. (2014). Attentional capture by evaluative stimuli: Gain- and loss-connoting colors boost the additional-singleton effect. Psychonomic Bulletin \& Review, 21(3), 701-707. https://doi.org/10.3758/s13423-013-0531-z.

Wiers, R. W., Rinck, M., Dictus, M., \& Van den Wildenberg, E. (2009). Relatively strong automatic appetitive action-tendencies in male carriers of the OPRM1 G-allele. Genes, Brain and Behavior, 8(1), 101-106. https://doi.org/10.1111/j.1601-183X.2008.00454.x.

Wirth, R., Dignath, D., Pfister, R., Kunde, W., \& Eder, A. B. (2016). Attracted by rewards: Disentangling the motivational influence of rewarding and punishing targets and distractors. Motivation Science, 2(3), 143-156. https://doi.org/10.1037/mot0000037.

Zajonc, R. B. (1980). Feeling and thinking: Preferences need no inferences. American Psychologist, 35(2), 151-175. https://doi. org/10.1037/0003-066X.35.2.151. 